\title{
FOURIER EXPANSIONS OF MODULAR FORMS AND PROBLEMS OF PARTITION ${ }^{1}$
}

\author{
HANS RADEMACHER
}

The subject which I am going to discuss in this lecture excels in the richness of its ramifications and in the diversity of its relations to other mathematical topics. I think therefore that it will serve our present purpose better not to attempt a systematic treatment, beginning with definitions and proceeding to lemmas, theorems, and proofs, but rather to look around and to envisage some outstanding marks scattered in various directions. I hope that the intrinsic relationships connecting the problems and theorems which I shall mention will nevertheless remain quite visible.

A good deal of the investigations about which I shall report can be subsumed under the heading of analytic number theory, and, more specifically, analytic additive number theory. It would, however, be a misplacement of emphasis if we were to look upon analysis, which here means function theory, only as a tool applied to the investigation of number theory. It is more the inner harmony of a system which I wish to depict, properties of functions revealing the nature of certain arithmetical facts, and properties of numbers having a bearing on the character of analytic functions.

Whereas the multiplicative number theory, which deals with questions of factorization, divisibility, prime numbers, and so on, goes back more than 2000 years to Euclid, the history of additive number theory, in any noteworthy sense, begins with Euler less than 200 years ago. In his famous treatise, Introductio in Analysin Infinitorum (1748), Euler devotes the sixteenth chapter, "De partitione numerorum," to problems of additive number theory. A "partition" is, after Euler, a decomposition of a natural number into summands which are natural numbers, for example, $6=1+1+4$. We can impose various restrictions on the summands; they may belong to a specified class of numbers, let us say odd numbers, or squares, or cubes, or primes; it may be required that they be all different; or their number may be preassigned. I wish to speak here only about unrestricted partitions. By the way, only the parts are essential, not their arrangement, so that we do not count two decompositions as different if they differ only in the order of the summands; we can therefore take the summands ordered according to their size.

${ }^{1}$ An address delivered before the Williamsburg meeting of the Society, December 29, 1938, by invitation of the Program Committee. 
The first step in any characterization will be to count the partitions. Thus we have the following 11 partitions of 6 :

$$
6,1+5,2+4,3+3,1+1+4,1+2+3,2+2+2,
$$

$1+1+1+3,1+1+2+2,1+1+1+1+2,1+1+1+1+1+1$.

We write $p(6)=11$, and in general denote the number of partitions of $n$ by $p(n)$.

As far as the method is concerned, Euler made the simple remark that, since we have $x^{m} \cdot x^{n}=x^{m+n}$, exponents of powers can easily be combined in an additive manner, and therefore products of power series can be used as "generating functions." In our case, for the unrestricted partitions, he found by simple reasoning the generating function

$$
f(x)=1+\sum_{n=1}^{\infty} p(n) x^{n}=\frac{1}{(1-x)\left(1-x^{2}\right)\left(1-x^{3}\right) \cdots} .
$$

Indeed, in a partition of $n$ we can first collect the equal summands and thereby express $n$ as a sum of multiples of $1,2,3, \cdots$,

$$
n=m_{1} \cdot 1+m_{2} \cdot 2+m_{3} \cdot 3+\cdots, \quad m_{j} \geqq 0 .
$$

On the other side

$$
\frac{1}{(1-x)\left(1-x^{2}\right)\left(1-x^{3}\right) \cdots}=\sum_{m_{1}=0}^{\infty} x^{m_{1}} \cdot \sum_{m_{2}=0}^{\infty} x^{m_{2} \cdot 2} \cdot \sum_{m_{3}=0}^{\infty} x^{m_{3} \cdot 3} \cdots,
$$

and the power $x^{n}$ therefore occurs as often in the product as $n$ can be written in the form (2); but the number of solutions of the diophantine equation (2) is precisely $p(n)$.

Euler later investigated the infinite product

$$
P(x)=(1-x)\left(1-x^{2}\right)\left(1-x^{3}\right) \cdots
$$

appearing in the right member of (1). He found, first empirically ${ }^{2}$ and later with conclusive proof, ${ }^{3}$ that

(3b) $P(x)=1-x-x^{2}+x^{5}+x^{7}--++=\sum_{\lambda=-\infty}^{\infty}(-1)^{\lambda} x^{\lambda(3 \lambda-1) / 2}$.

The discovery of the equality expressed by (3a) and (3b) marks a

${ }^{2}$ Découverte d'une loi tout extraordinaire des nombres par rapport à la somme de leurs diviseurs, Opera Omnia, (1), vol. 2, pp. 241-253.

${ }^{3}$ Demonstratio theorematis circa ordinem in summis divisorum observatum (17541755), Opera Omnia, (1), vol. 2, pp. 390-398). 
highly important event in the history of our science. It is the first time that a $\vartheta$-function, in a special case, appears in the literature; moreover it appears here immediately in its two aspects: as a power series with exponents formed by a quadratic expression of the index of summation, and secondly as an infinite product. ${ }^{4}$ This equation leads to the identity

$$
\left(1+\sum_{n=1}^{\infty} p(n) x^{n}\right) \sum_{\lambda=-\infty}^{\infty}(-1)^{\lambda} x^{\lambda(3 \lambda-1) / 2}=1
$$

which furnishes a formula of recurrence for $p(n)$. Formulas of recurrence have been used, indeed, for the construction of tables of $p(n)$ for values of $n$ up to 600 (MacMahon, ${ }^{5} \mathrm{Gupta}^{6}$ ). The function $p(n)$ is very rapidly increasing with $n$. Some specimens are

$$
\begin{gathered}
p(10)=42, \quad p(100)=190569292, \quad p(200)=3972999029388, \\
p(600)=458004788008144308553622 .
\end{gathered}
$$

Outside the range from 1 to 600 a few isolated values of $p(n)$ have been computed by D. H. Lehmer. ${ }^{7}$ That, of course, could not be done by a formula of recurrence, but has been made possible by certain independent representations of $p(n)$, which belong to the main part of this report. Let me mention only that the largest known value of $p(n)$ is $p(14031)$, which turns out to be a figure of 127 digits.

In spite of the profound discoveries in the field of $\vartheta$-functions and related functions due to Jacobi, Riemann, Klein, and Poincaré, the situation of our problem remained unchanged for more than one and a half centuries after Euler's investigations. It was not until 1917 that

${ }^{4}$ In a letter to Fuss, the first editor of Euler's unpublished works, Jacobi writes (1848), "Ich möchte mir bei dieser Gelegenheit noch erlauben, Ihnen zu sagen, warum ich mich so sehr für diese EULERsche Entdeckung interessiere. Sie ist nämlich der erste Fall gewesen, in welchem Reihen aufgetreten sind, deren Exponenten eine arithmetische Reihe zweiter Ordnung bilden, und auf diese Reihen ist durch mich die Theorie der elliptischen Transcendenten gegründet worden. Die EULERsche Formel ist ein spezieller Fall einer Formel, welche wohl das wichtigste und fruchtbarste ist, was ich in reiner Mathematik erfunden habe..." (quoted from Euler, Opera Omnia, (1), vol. 2, p. 192, footnote).

B This table was published by Hardy and Ramanujan in the paper referred to in footnote 8 .

${ }^{6} \mathrm{~A}$ table of partitions, Proceedings of the London Mathematical Society, (2), vol. 39 (1935), pp. 142-149; A table of partitions II, Proceedings of the London Mathematical Society, (2), vol. 42 (1937), pp. 546-549.

${ }^{7}$ On a conjecture of Ramanujan, Journal of the London Mathematical Society, vol. 11 (1936), pp. 114-118. An application of Schläfi's modular equation to a conjecture of Ramanujan, this Bulletin, vol. 44 (1938), pp. 84-90. 
G. H. Hardy and S. Ramanujan published their fundamental paper, A symptotic formulae in combinatory analysis. ${ }^{8}$ As I have already mentioned, the denominator in (1) is closely related to $\vartheta$-functions. In a commentary on Riemann's Collected Works, and in another paper, ${ }^{9}$ Dedekind had made detailed studies of the function

$$
\eta(\tau)=e^{\pi i \tau / 12} \prod_{m=1}^{\infty}\left(1-e^{2 \pi i m r}\right), \quad \Im(\tau)>0,
$$

which, under the substitution $x=e^{2 \pi i \tau}$, is essentially the denominator in question.

Hardy and Ramanujan started with Cauchy's formula applied to the equation (1):

$$
p(n)=\frac{1}{2 \pi i} \int_{C} \frac{f(x)}{x^{n+1}} d x .
$$

Since $f(x)$ is regular inside the unit circle and has the unit circle as its natural boundary, $C$ has to be a closed curve inside $|x|=1$, surrounding the point $x=0$.

Now the usual approach to a complex integral is to utilize the freedom in the path of integration, whether we use the calculus of residues or the method of steepest descents or similar devices. In any case we trace the path of integration in such a way that it passes through that region in which the function, by the overwhelming amount of its value, gives the heaviest contribution on a relatively short piece of the path. The function $f(x)=P(x)^{-1}$ tends rapidly to infinity if we approach radially the point $x=1$, since each term in $P(x)$ tends to zero. Thus the neighborhood of $x=1$ will yield the most essential contribution. The next heaviest singularity is located at $x=-1$ in whose vicinity every other factor of $P(x)$ comes close to zero. In this way the roots of unity enter, according to their denominators, but decreasing in weight with increasing denominators. The path $C$ is taken as a circle around 0 rather close to the unit circle, and we cut it into parts, each part corresponding to a neighborhood of just one root of unity. The assembly of all proper fractions $h / k$ with $k \leqq N$ is called the Farey series of order $N$, to the use of which Hardy and Ramanujan were quite naturally led by these considerations.

${ }^{8}$ Asymptotic formulae in combinatory analysis, Proceedings of the London Mathematical Society, (2), vol. 17 (1918), pp. 75-115.

' Schreiben an Herrn Borchardt über die Theorie der elliptischen Modulfunktionen, Journal für die reine und angewandte Mathematik, vol. 83 (1877), pp. 265-292; also Gesammelte Werke, vol. 1, pp. 175-201. 
This is, of course, only a sketch of the procedure of integration. Its details have to be furnished by a study of the function $f(x)$ in the neighborhood of a root of unity $e^{2 \pi i h / k}$. This is given by the formula

$$
\begin{array}{r}
f\left(e^{2 \pi i h / k-2 \pi z / k}\right)=\omega_{h, k} z^{1 / 2} \exp \left(\frac{\pi}{12 k z}-\frac{\pi z}{12 k}\right) f\left(e^{2 \pi i h^{\prime} / k-2 \pi / k z}\right), \\
\Re(z)>0 .
\end{array}
$$

Here $\omega_{h, k}$ is a certain $24 k$ th root of unity. This formula is of importance in our problem since in the neighborhood of the root of unity $e^{2 \pi i h / k}$ we have $z$ small, $1 / z$ large, and both $z$ and $1 / z$ with positive real parts. Such a large $1 / z$ involves a very small value of $e^{2 \pi i h^{\prime} / k-2 \pi / k z}$ so that, because of (1), $f\left(e^{2 \pi i h^{\prime} / k-2 \pi / k z}\right)$ can with good approximation (which has, of course, to be appraised) be replaced by 1 . This gives an elementary approximation function for $f(x)$ in the neighborhood of $e^{2 \pi i h / k}$. By means of this treatment of (5), and appropriate estimations of the errors which are made when the function $f(x)$ is replaced by certain approximation functions, Hardy and Ramanujan arrived at the asymptotic formula

$$
\begin{aligned}
p(n)= & \frac{1}{2 \pi 2^{1 / 2}} \sum_{k \leqq \alpha n^{1 / 2}} A_{k}(n) k^{1 / 2} \frac{d}{d n}\left(\frac{\exp \left\{\frac{\pi}{k}\left[\frac{2}{3}(n-1 / 24)\right]^{1 / 2}\right\}}{(n-1 / 24)^{1 / 2}}\right) \\
& +O\left(n^{-1 / 4}\right),
\end{aligned}
$$

with

$$
A_{k}(n)=\sum_{h \bmod } \omega_{k,(h, k)=1} \omega_{h, k} e^{-2 \pi i n h / k} .
$$

This formula is remarkable in analytic number theory because of its error term which tends to zero as $n$ increases. The constant involved in the error term was not determined; actual computation of $p(100)$ and $p(200)$, however, showed that a relatively small number of terms suffice to give a value which differs from the true value by only a few thousandths of a unit. Hardy and Ramanujan raised the question whether the series (7) extended indefinitely converges or not. D. H. Lehmer ${ }^{10}$ has shown recently, by a study of the $A_{k}(n)$, that the infinite series (7) is divergent.

The method applied by Hardy and Ramanujan was further developed by Hardy and Littlewood and applied to other problems, in particular to Waring's problem, where it leads also to asymptotic

${ }^{10}$ On the Hardy-Ramanujan series for the partition function, Journal of the London Mathematical Society, vol. 12 (1937), pp. 171-176. 
results. In the present case, however, Hardy and Ramanujan were not aware of the full strength of their method. When it is applied with more refinement of the estimates, and when in particular the coupling of $N$ (the order of the Farey dissection) with $n$ (the given integer) is abolished, and $N$ is made to go to infinity with a fixed $n$, then we obtain the following exact formula ${ }^{11}$ for $p(n)$ :

$$
p(n)=\frac{1}{\pi 2^{1 / 2}} \sum_{k=1}^{\infty} A_{k}(n) k^{1 / 2} \frac{d}{d n}\left(\frac{\sinh \frac{\pi}{k}\left[\frac{2}{3}(n-1 / 24)\right]^{1 / 2}}{(n-1 / 24)^{1 / 2}}\right)
$$

where the infinite series is absolutely convergent. The formula (7) not only appears as a consequence of formula (9), but also the error term in (7) can be definitely estimated. This, by the way, made it possible to compute the function $p(n)$ for the great values of $n$ mentioned above.

The formula (9) has now been the starting point for further results. We saw that the formula (6) forms the basis for the evaluation of the integral (1). The formula (6) in turn is derived from the theory of the function $\eta(\tau)$. We have

$$
\begin{gathered}
\eta\left(\tau^{\prime}\right)=\epsilon(a, b, c, d)[-i(c \tau+d)]^{1 / 2} \eta(\tau), \quad c>0 \\
\tau^{\prime}=\frac{a \tau+b}{c \tau+d}, \quad\left|\begin{array}{ll}
a & b \\
c & d
\end{array}\right|=1,
\end{gathered}
$$

where $a, b, c, d$ are integers and $\epsilon$ is a certain 24 th root of unity. This formula is connected with (6) through the substitution

$$
\tau=\frac{i z+h}{k}, \quad \tau^{\prime}=\frac{i / z+h^{\prime}}{k}, \quad h h^{\prime} \equiv-1(\bmod k) .
$$

The substitutions (10a), together with the case $c=0$ :

$$
\tau^{\prime}=\tau+b,
$$

for which we have, directly from (4),

$$
\eta\left(\tau^{\prime}\right)=e^{\pi \dot{i} b / 12} \eta(\tau)
$$

are called modular substitutions. They form an infinite discontinuous

${ }^{11}$ Rademacher, $A$ convergent series for the partition function $p(n)$, Proceedings of the National Academy of Sciences, vol. 23 (1937), pp. 78-84; On the partition function $p(n)$, Proceedings of the London Mathematical Society, (2), vol. 43 (1937), pp. 241-254. 
group. All these considerations can be applied to modular forms in general. ${ }^{12}$ Let us give, in brief, a definition of this concept.

A modular form is a homogeneous analytic function of two variables $\omega_{1}, \omega_{2}$ defined for $\Im\left(\omega_{2} / \omega_{1}\right)>0$ :

$$
H\left(\lambda \omega_{1}, \lambda \omega_{2}\right)=\lambda^{r} H\left(\omega_{1}, \omega_{2}\right) ;
$$

the parameter $r$, which we take as real, is the dimension of the form. A modular form is invariant under modular transformations :

$$
\begin{aligned}
& \begin{array}{l}
\omega_{2}^{\prime}=a \omega_{2}+b \omega_{1}, \\
\omega_{1}^{\prime}=c \omega_{2}+d \omega_{1},
\end{array}\left|\begin{array}{ll}
a & b \\
c & d
\end{array}\right|=1, \\
& H\left(\omega_{1}^{\prime}, \omega_{2}^{\prime}\right)=H\left(\omega_{1}, \omega_{2}\right) \text {. }
\end{aligned}
$$

Examples are the invariants

$$
g_{2}=60 \sum^{\prime} \frac{1}{\left(2 m_{1} \omega_{1}+2 m_{2} \omega_{2}\right)^{4}}, \quad g_{3}=140 \sum^{\prime} \frac{1}{\left(2 m_{1} \omega_{1}+2 m_{2} \omega_{2}\right)^{6}} .
$$

A nonhomogeneous notation is often preferable: By means of (11) we have

$$
\omega_{1}^{\prime} H\left(1, \omega_{2}^{\prime} / \omega_{1}^{\prime}\right)=\omega_{1}^{r} H\left(1, \omega_{2} / \omega_{1}\right)
$$

and, with $\omega_{2} / \omega_{1}=\tau, \omega_{2}^{\prime} / \omega_{1}^{\prime}=\tau^{\prime}, \tau^{\prime}=(a \tau+b) /(c \tau+d)$,

$$
H\left(1, \tau^{\prime}\right)=(c \tau+d)^{-r} H(1, \tau) .
$$

If $r$ is not an integer, we have to determine the branch of $(c \tau+d)^{-r}$. In order to avoid this difficulty we admit a slight generalization. We consider functions $F(\tau)$ for $\Im(\tau)>0$ with the property

$$
F\left(\tau^{\prime}\right)=\epsilon(a, b, c, d) \cdot(-i(c \tau+d))^{-r} F(\tau),
$$

where $c>0$ and $|\epsilon|=1$ and $(-i(c \tau+d))^{-r}$ stands for the principal branch. The case $c=0$ has to be mentioned separately:

$$
F(\tau+1)=\epsilon_{1} F(\tau)=e^{2 \pi i \alpha} F(\tau), \quad 0 \leqq \alpha<1 .
$$

A function $F(\tau)$ having the properties (13a) and (13b) may now be called a "modular form," in spite of its nonhomogeneous notation; in particular (13a) shows that it is of dimension $r$.

From (13b) we derive

$$
e^{-2 \pi i \alpha(\tau+1)} F(\tau+1)=e^{-2 \pi i \alpha \tau} F(\tau),
$$

12 Rademacher and Zuckerman, On the Fourier coefficients of certain modular forms of positive dimension, Annals of Mathematics, (2), vol. 39 (1938), pp. 433-462. 
and note the periodicity in $\tau$ modulo 1 . Therefore we have a Fourier development

$$
F(\tau)=e^{2 \pi i \alpha \tau} \sum_{n=-\infty}^{\infty} a_{n} e^{2 \pi i n \tau},
$$

which will converge in the whole upper half-plane if $F(\tau)$ is assumed to be regular there. We then call $F(\tau)$ an "entire" modular form. One further restriction is important: we assume that (14) contains only a finite number of terms with negative exponents, or, as we can say, $F(\tau)$ has a pole at the parabolic point $\tau=i \infty$ measured in the uniformizing variable $e^{2 \pi i \tau}$.

In our previous case we had

$$
\eta(\tau)^{-1}=e^{2 \pi i(23 / 24) \tau} \sum_{n=-1}^{\infty} p(n+1) e^{2 \pi i n \tau} .
$$

The partition function appears here therefore as the Fourier coefficients of a modular function of dimension $+1 / 2$ (since $\eta(\tau)$ in (10) has the dimension $-1 / 2$ ). Our method now enables us to determine the coefficients $a_{n}$ for $n \geqq 0$ from the principal part of the pole at the parabolic point $\tau=i \infty$, that is, from the coefficients $a_{n}$ with $n<0$.

I cannot go into details of the application of the Hardy-Ramanujan-Littlewood method. Only one essential point need be mentioned: it is important that $r$ be positive. Indeed, this $r$ is responsible for a term $z^{r}$ corresponding to $z^{1 / 2}$ in (6), and, with $z$ approaching zero, helps in a decisive way to ensure convergence.

The result is this:

If $F(\tau)$ is an entire modular form of positive dimension $r$,

$$
\begin{array}{rlrl}
F\left(\frac{a \tau+b}{c \tau+d}\right) & =\epsilon(a, b, c, d)(-i(c \tau+d))^{-r} F(\tau), & c>0, \\
F(\tau+1) & =e^{2 \pi i \alpha} F(\tau), & & 0 \leqq \alpha<1, \\
F(\tau) & =e^{2 \pi i \alpha \tau} \sum_{m=-\mu}^{\infty} a_{m} e^{2 \pi i m \tau}, &
\end{array}
$$

then

$$
\begin{aligned}
a_{m}=\sum_{\nu=1}^{\mu} a_{-\nu} \sum_{k=1}^{\infty} \frac{1}{k} A_{k, \nu}(m)\left(\frac{\nu-\alpha}{m+\alpha}\right)^{(r+1) / 2} \\
\cdot I_{r+1}\left((4 \pi / k)[(\nu-\alpha)(m+\alpha)]^{1 / 2}\right), \quad m \geqq 0,
\end{aligned}
$$




$$
\begin{aligned}
A_{k, \nu}(m)= & \sum_{0 \leqq h<k,(h, k)=1} \epsilon\left(h^{\prime},-\left(h h^{\prime}+1\right) / k, k,-h\right)^{-1} \\
& \cdot \exp \left\{-(2 \pi i / k)\left((\nu-\alpha) h^{\prime}+(m+\alpha) h\right)\right\} .
\end{aligned}
$$

Before I come to consequences of these equations for coefficients, I wish to mention two important extensions. We have taken into consideration only modular forms invariant with respect to the full modular group

$$
\tau^{\prime}=\frac{a \tau+b}{c \tau+d}, \quad\left|\begin{array}{ll}
a & b \\
c & d
\end{array}\right|=1 .
$$

But we can just as well consider a subgroup of the full modular group. It is only necessary that its fundamental region have a finite number of parabolic points. The most important and best known subgroups of this sort are the congruence subgroups, that is, those in which $a, b, c, d$ are subjected to certain congruence restrictions. The principal congruence subgroup modulo $p$ is that which has

$$
\left(\begin{array}{ll}
a & b \\
c & d
\end{array}\right) \equiv\left(\begin{array}{ll}
1 & 0 \\
0 & 1
\end{array}\right) \quad(\bmod p) .
$$

Zuckerman ${ }^{13}$ has carried out this generalization.

Secondly we can overcome to a certain extent the restriction that the dimension must be positive. For this purpose we have to be more careful with our estimates. The sums $A_{k, \nu}(m)$ can immediately be estimated as $\left|A_{k, v}(m)\right| \leqq \sum 1=\phi(k)$. The problem of a better estimate has not been fully solved. But in certain simple cases we can get

$$
A_{k, \nu}(m)=O\left(k^{2 / 3+\epsilon}\right) \text {. }
$$

These improved estimates were first begun by Kloosterman ${ }^{14}$ after whom these sums are now named, and later continued by Esterman, ${ }^{15}$ Salié, ${ }^{16}$ Davenport ${ }^{17}$ Lehmer, ${ }^{18}$ and others. In this way we can easily

${ }^{13}$ On the coefficients of certain modular forms belonging to subgroups of the modular group, Transactions of this Society, vol. 45 (1939), pp. 298-321.

${ }_{14}$ Asymptotische Formeln für die Fourierkoeffizienten ganzer Modulformen, Abhandlungen aus dem mathematischen Seminar der Hamburgischen Universität, vol. 5 (1927), pp. 337-352.

15 Vereinfachter Beweis eines Satzes von Kloosterman, Abhandlungen aus dem mathematischen Seminar der Hamburgischen Universität, vol. 7 (1939), pp. 82-98.

${ }^{16}$ Zur Abschätzung der Fourierkoeffizienten ganzer Modulformen, Mathematische Zeitschrift, vol. 36 (1933), pp. 263-278.

17 On certain exponential sums, Journal für die reine und angewandte Mathematik, vol. 169 (1933), pp. 158-176.

18 On the series for the partition function, Transactions of this Society, vol. 43 (1938), pp. 271-295. 
include the case with dimension zero in our reasonings. A very important function belongs to that class, the modular invariant $J(\tau)$ :

$$
\begin{gathered}
J(\tau)=\frac{g_{2}{ }^{3}}{\Delta}=\frac{g_{2}{ }^{3}}{g_{2}{ }^{3}-27 g_{3}{ }^{2}}, \quad J\left(\frac{a \tau+b}{c \tau+d}\right)=J(\tau), \\
12^{3} J(\tau)=e^{-2 \pi i \tau}+c_{0}+\sum_{n=1}^{\infty} c_{n} e^{2 \pi i n \tau} .
\end{gathered}
$$

The coefficients $c_{n}$ of this fundamental function can be found by our method $^{19}$ as

$$
\begin{aligned}
c_{n} & =\frac{2 \pi}{n^{1 / 2}} \sum_{k=1}^{\infty} \frac{1}{k} A_{k}(n) I_{1}\left((4 \pi / k) n^{1 / 2}\right), \quad n \geqq 1, \\
A_{k}(n) & =\sum_{h \bmod k,(h, k)=1} e^{-(2 \pi i / k)\left(n h+h^{\prime}\right)} .
\end{aligned}
$$

It is of interest that a few years ago Petersson ${ }^{20}$ found these coefficients (which are integers) by a completely different method, which operates with modular functions of negative dimensions. Indeed, $J^{\prime}(\tau)$ is of dimension -2 as is readily seen,

$$
J^{\prime}\left(\frac{a \tau+b}{c \tau+d}\right) \frac{1}{(c \tau+d)^{2}}=J^{\prime}(\tau)
$$

from the differentiation of (17). Modular forms of negative dimension have, however, been studied as far back as Eisenstein, who found the partial fraction series for $g_{2}$ and $g_{3}$, and later by Poincaré among others. These investigations lie outside the field of our present discussion.

If I have in this way outlined definitions, methods, and direct results, I wish now to survey briefly a few consequences of our theory and some remaining problems. Let me begin with function theoretical consequences. Looking at formula (15), we see that the $a_{m}(m \geqq 0)$ depend linearly on $a_{-\mu}, \cdots, a_{-1}$. If all these should happen to be equal to zero, our analysis would go through just as well and would lead to $a_{m}=0$ for $m \geqq 0$. Hence we have that an entire modular form of positive dimension which is regular also at the parabolic point (or points) of the fundamental region vanishes identically. This is remarkable in so far as it is not true for modular forms of negative

${ }^{19}$ Rademacher, The Fourier coefficients of the modular invariant $J(\tau)$, American Journal of Mathematics, vol. 60 (1938), pp. 501-512.

${ }^{20}$ Ueber die Entwicklungskoeffizienten der automorphen Formen, Acta Mathematica, vol. 58 (1932), pp. 169-215. 
dimension, as for example $g_{2}(1, \tau), g_{3}(1, \tau), \vartheta_{3}(0 \mid \tau)$, and $\eta(\tau)$ show.

These facts have a bearing on the generalized Kloosterman sums, which occur in the estimates. For the case of $\eta(\tau)^{-1}$ we know

$$
A_{k}(n)=O\left(k^{1 / 2+\epsilon}\right),
$$

and for $J(\tau)$ (which leads to the original Kloosterman sums)

$$
A_{k}(n)=O\left(k^{2 / 3+\epsilon} n^{1 / 3}\right),
$$

after Salié and Davenport. It is highly probable that in the latter case a sharper estimate like (18a) will be true. However, our reasonings show that such estimates cannot be expected in all cases. Indeed, if there existed a positive constant $\alpha$ such that for all Kloosterman sums belonging to all modular forms we should have

$$
A_{k, \nu}(n)=O\left(k^{1-\alpha}\right),
$$

it would be possible to repeat our investigations of the coefficients, for example, for $\eta(\tau)^{\alpha / 2}$. Since this function (of dimension $-\alpha / 4$ ) is regular at infinity, the estimate $(18 \mathrm{~b})$ would imply the absurd consequence that all coefficients of $\eta(\tau)^{\alpha / 2}$ vanish identically. An estimate better than the trivial $O(k)$ for the generalized Kloosterman sums can therefore be obtained only under certain special conditions and not uniformly for all real dimensions.

We can use the exact coefficients which we have found again in our Fourier series. We have

$$
12^{3} J(\tau)=e^{-2 \pi i \tau}+c_{0}+\sum_{n=1}^{\infty} \frac{2 \pi}{n^{1 / 2}} e^{2 \pi i n \tau} \sum_{k=1}^{\infty} \frac{1}{k} A_{k}(n) I_{1}\left(4 \pi n^{1 / 2} / k\right) .
$$

This series, on the other hand, determines the function directly. It is clear that it satisfies the relation $J(\tau+1)=J(\tau)$. But it must also be invariant with respect to all other modular substitutions. Since all of them are generated by $\tau^{\prime}=\tau+1, \tau^{\prime}=-1 / \tau$, it is of interest to show directly $J(-1 / \tau)=J(\tau)$ by means of the series, or, in other words, to show that the series defines a modular function. This, indeed, can be done, as I have shown in a recent paper. ${ }^{21}$ The proof consists of a rearrangement of terms of a conditionally convergent double series.

I hope that this proof has a prospect of further development for the problem of existence. Up to the present we have only discussed modular forms of positive dimension which are given by certain other definitions (infinite products and so on). But the problem is to con-

${ }^{21}$ The Fourier series and the functional equation of the absolute modular invariant $J(\tau)$, American Journal of Mathematics, vol. 61 (1939), pp. 237-248. 
struct new ones with given principal parts at the parabolic points. For negative dimensions we have a powerful principle of generation in the Eisenstein-Poincaré series, which have no analogue here for reasons of convergence.

The exact formulas for the coefficients lead to a sort of analytic continuation which I wish to mention at least briefly. I exemplify this idea with the treatment of $f(x)=1+\sum_{n=1}^{\infty} p(n) x^{n}$. Introducing our value for $p(n)$ we obtain

$$
\begin{aligned}
& f(x)= 1+\frac{1}{\pi 2^{1 / 2}} \sum_{n=1}^{\infty} x^{n} \sum_{k=1}^{\infty} \sum_{h \bmod } \omega_{k,(h, k)=1} \omega_{h, k} e^{-2 \pi i h n / k} \\
& \cdot k^{1 / 2} \frac{d}{d n}\left(\frac{\sinh \frac{\pi}{k}\left[\frac{2}{3}(n-1 / 24)\right]^{1 / 2}}{(n-1 / 24)^{1 / 2}}\right) \\
&=1+\frac{1}{\pi 2^{1 / 2}} \sum_{k=1}^{\infty} \sum_{h \bmod k,(h, k)=1} \omega_{h, k} k^{1 / 2} \sum_{n=1}^{\infty}\left(x e^{-2 \pi i h / k}\right)^{n} \\
& \cdot \frac{d}{d n}\left(\frac{\sinh \frac{\pi}{k}\left[\frac{2}{3}(n-1 / 24)\right]^{1 / 2}}{(n-1 / 24)^{1 / 2}}\right) \\
&= 1+\frac{1}{\pi 2^{1 / 2}} \sum_{h \bmod k,(h, k)=1} \omega_{h, k} k^{1 / 2} \Phi_{k}\left(x e^{-2 \pi i h / k}\right),
\end{aligned}
$$

with

(20a) $\Phi_{k}(z)=\sum_{n=1}^{\infty} \frac{d}{d n}\left(\frac{\sinh \frac{\pi}{k}\left[\frac{2}{3}(n-1 / 24)\right]^{1 / 2}}{(n-1 / 24)^{1 / 2}}\right) z^{n}=\sum_{n=1}^{\infty} g_{k}(n) z^{n}$.

Here

$$
\begin{aligned}
g_{k}(n) & =\frac{d}{d n}\left(\frac{\sinh \frac{\pi}{k}\left[\frac{2}{3}(n-1 / 24)\right]^{1 / 2}}{(n-1 / 24)^{1 / 2}}\right) \\
& =\frac{d}{d n} \sum_{\nu=0}^{\infty} \frac{\left[(\pi / k)(2 / 3)^{1 / 2}\right]^{2 \nu+1}}{(2 \nu+1) !}(n-1 / 24)^{\nu} \\
& =\sum_{\nu=1}^{\infty}\left[(\pi / k)(2 / 3)^{1 / 2}\right]^{2 \nu+1} \frac{\nu}{(2 \nu+1) !}(n-1 / 24)^{\nu-1}
\end{aligned}
$$


is a transcendental function of order $1 / 2$ in the variable $n$. Hence by a theorem of Wigert $\Phi_{k}(z)$, which originally is defined only in $|z|<1$, can be extended over the whole z-plane and has only an isolated essential singularity at $z=1$. The formula (20) therefore represents the splitting up of $f(x)$ into functions each having just one essential singularity. All singularities together form the natural boundary $|x|=1$.

Moreover, the series (20) has now a meaning for $|x|>1$. It turns out that it converges there also. Simple considerations show that, for $|z|>1$, we get

$$
\Phi_{k}(z)=\sum_{m=0}^{\infty} \frac{d}{d m}\left(\frac{\sin \frac{\pi}{k}\left[\frac{2}{3}(m+1 / 24)\right]^{1 / 2}}{(m+1 / 24)^{1 / 2}}\right) z^{-m}
$$

and so, for $|x|>1$, if we introduce $f^{*}(x)$ instead of $f(x)$,

$$
\begin{aligned}
f^{*}(x)= & 1+\frac{1}{\pi 2^{1 / 2}} \sum_{h \bmod k,(h, k)=1} \omega_{h, k} k^{1 / 2} \\
& \cdot \sum_{m=0}^{\infty} \frac{d}{d m}\left(\frac{\left.\sin \frac{\pi}{k}\left[\frac{2}{3}(m+1 / 24)\right]^{1 / 2}\right)}{(m+1 / 24)^{1 / 2}}\right) \cdot\left(x e^{-2 \pi i h / k}\right)^{-m} \\
= & 1+\sum_{m=0}^{\infty} p^{*}(m) x^{-m}
\end{aligned}
$$

let us say. Here

$$
\begin{aligned}
p^{*}(m) & =\frac{1}{\pi 2^{1 / 2}} \sum_{k=1}^{\infty} A_{k}(-m) k^{1 / 2} \frac{d}{d m}\left(\frac{\sin \frac{\pi}{k}\left[\frac{2}{3}(m+1 / 24)\right]^{1 / 2}}{(m+1 / 24)^{1 / 2}}\right) \\
& =-p(-m),
\end{aligned}
$$

formally. Now $f^{*}(x)=0$ identically. Indeed, it turns out that we get $p(0)=1$, as is to be expected, and $p(-m)=0$ for $m \geqq 1$. This was first proved by Petersson ${ }^{22}$ in a recent publication, making use again of modular forms of negative dimension. It can, however, also be proved by means of the Hardy-Ramanujan method.

Similar "continuations" beyond the natural boundary can be effec-

${ }_{22}^{2}$ Die linearen Relationen zwischen den ganzen Poincaréschen Reihen von reeller Dimension zur Modulgruppe, Abhandlungen aus dem mathematischen Seminar der Hamburgischen Universität, vol. 12 (1938), pp. 415-472. 
tuated for all those power series arising from modular forms of nonnegative dimension. For $J(\tau)$ we obtain, for example,

$$
J(\tau)=F\left(e^{2 \pi i \tau}\right)=F(x), \quad|x|<1,
$$

with $F^{*}(x)=5 / 12,(|x|>1)$.

I come finally to some arithmetical consequences of the method connected mainly with partitions. If we admit only odd integers as parts, then the generating function for the number $q(n)$ of partitions into odd summands is

$$
\begin{aligned}
g(x) & =1+\sum_{n=1}^{\infty} q(n) x^{n}=\frac{1}{(1-x)\left(1-x^{3}\right)\left(1-x^{5}\right) \cdots} \\
& =\frac{\left(1-x^{2}\right)\left(1-x^{4}\right) \cdots}{(1-x)\left(1-x^{2}\right) \cdots}=\frac{f(x)}{f\left(x^{2}\right)} .
\end{aligned}
$$

This can be treated by our method, but with the difficulty that we have to deal with modular forms of dimension zero and belonging to a congruence subgroup modulo 2 . This has been done by Hua in an unpublished paper. The modular form here would be $\eta(2 \tau) / \eta(\tau)$. As a result Hua obtains again a convergent series for $q(n)$, of which Hardy and Ramanujan had given only the first few terms.

There are a number of problems of this sort concerning restricted partitions, which can be attacked by our method. For example Niven $^{23}$ has recently determined the number of partitions of a number into summands of the form $6 n \pm 1$.

A further consequence which seems more interesting to me is the following: Ramanujan discovered, first empirically, the properties

$$
\begin{aligned}
p(5 m+4) & \equiv 0(\bmod 5), \\
p(7 m+5) & \equiv 0(\bmod 7), \\
p(11 m+6) & \equiv 0(\bmod 11) .
\end{aligned}
$$

He was able to prove (a) and (b) easily but remarked that they would be obvious consequences of the identities

$$
\begin{aligned}
\sum_{m=0}^{\infty} p(5 m+4) x^{m} & =5 \frac{\Pi\left(1-x^{5 \nu}\right)^{5}}{\Pi\left(1-x^{\nu}\right)^{6}}, \\
\sum_{m=0}^{\infty} p(7 m+5) x^{m} & =7 \frac{\prod\left(1-x^{7 \nu}\right)^{3}}{\prod\left(1-x^{\nu}\right)^{4}}+49 x \frac{\Pi\left(1-x^{7 \nu}\right)^{7}}{\Pi\left(1-x^{\nu}\right)^{8}} .
\end{aligned}
$$

He did not give a proof of these identities. Proofs were later given

${ }^{23}$ On a certain partition function, to be published. 
by Darling ${ }^{24}$ and Mordell ${ }^{25}$ and recently again by Watson ${ }^{26}$ in a comprehensive paper which covers much more. Now our method can be directly applied to these identities. ${ }^{27}$ We know the coefficients of the left member and can express the right member in the same way. All we need to do is then to compare coefficients, which turns out to be not completely trivial since the expressions need a slight transformation.

Zuckerman ${ }^{28}$ has found a new identity of this sort by our method. If we write $\Pi\left(1-x^{\nu}\right)=\phi(x)$, then

$$
\begin{aligned}
\sum_{m=0}^{\infty} p(13 m+6) & x^{m}=11 \frac{\phi\left(x^{13}\right)}{\phi(x)^{2}}+36 \cdot 13 x \frac{\phi\left(x^{13}\right)^{3}}{\phi(x)^{4}}+38 \cdot 13^{2} x^{2} \frac{\phi\left(x^{13}\right)^{5}}{\phi(x)^{6}} \\
& +20 \cdot 13^{3} x^{3} \frac{\phi\left(x^{13}\right)^{7}}{\phi(x)^{8}}+6 \cdot 13^{4} x^{4} \frac{\phi\left(x^{13}\right)^{9}}{\phi(x)^{10}}+13^{5} x^{5} \frac{\phi\left(x^{13}\right)^{11}}{\phi(x)^{12}} \\
& +13^{5} x^{6} \frac{\phi\left(x^{13}\right)^{13}}{\phi(x)^{14}}
\end{aligned}
$$

It is regrettable that this identity does not lead to arithmetical properties of the Ramanujan sort since the factor 13 does not appear as a factor of every term of the right member.

The connection between modular functions and partitions seems to be accidental. Analogues of these concepts may be found in algebraic fields. However they are not connected as in the rational case by formulas of the type (1). Our method could probably be carried over to the discussion of these modular functions, but they would not yield any information concerning these partition functions.

\section{University of Pennsylvania}

${ }^{24}$ Proofs of certain identities and congruences enunciated by S. Ramanujan, Proceedings of the London Mathematical Society, (2), vol. 19 (1921), pp. 350-372.

${ }^{25}$ Note on certain modular relations considered by Messrs. Ramanujan, Darling, and Rogers, Proceedings of the London Mathematical Society, (2), vol. 20 (1922), pp. 408-416.

${ }^{26}$ Ramanujans Vermutung über Zerfällungsanzahlen, Journal für die reine und angewandte Mathematik, vol. 179 (1938), pp. 97-128.

${ }^{27}$ Rademacher and Zuckerman, $A$ new proof of two of Ramanujan's identities, Annals of Mathematics, (2), vol. 40 (1939), pp. 473-489.

${ }^{28}$ Identities analogous to Ramanujan's identities involving the partition function, Duke Mathematical Journal, vol. 5 (1939), pp. 88-110. 